Proceedings of the Conference on Empirical Methods in Natural

Language Processing (EMNLP), Philadelphia, July 2002, pp. 188-195.

Association for Computational Linguistics.

\title{
A Method for Open-Vocabulary Speech-Driven Text Retrieval
}

\author{
Atsushi Fujii* \\ University of Library and \\ Information Science \\ 1-2 Kasuga, Tsukuba \\ 305-8550, Japan \\ fujiculis.ac.jp
}

\author{
Katunobu Itou \\ National Institute of \\ Advanced Industrial \\ Science and Technology \\ 1-1-1 Chuuou Daini Umezono \\ Tsukuba, 305-8568, Japan \\ itou@ni.aist.go.jp
}

\author{
Tetsuya Ishikawa \\ University of Library and \\ Information Science \\ 1-2 Kasuga, Tsukuba \\ 305-8550, Japan \\ ishikawa@ulis.ac.jp
}

\begin{abstract}
While recent retrieval techniques do not limit the number of index terms, out-ofvocabulary (OOV) words are crucial in speech recognition. Aiming at retrieving information with spoken queries, we fill the gap between speech recognition and text retrieval in terms of the vocabulary size. Given a spoken query, we generate a transcription and detect OOV words through speech recognition. We then correspond detected OOV words to terms indexed in a target collection to complete the transcription, and search the collection for documents relevant to the completed transcription. We show the effectiveness of our method by way of experiments.
\end{abstract}

\section{Introduction}

Automatic speech recognition, which decodes human voice to generate transcriptions, has of late become a practical technology. It is feasible that speech recognition is used in real-world human language applications, such as information retrieval.

Initiated partially by TREC-6, various methods have been proposed for "spoken document retrieval (SDR)," in which written queries are used to search speech archives for relevant information (Garofolo et al., 1997). State-of-the-art SDR methods, where speech recognition error rate is $20-30 \%$, are

${ }^{*}$ The first and second authors are also members of CREST, Japan Science and Technology Corporation. comparable with text retrieval methods in performance (Jourlin et al., 2000), and thus are already practical. Possible rationales include that recognition errors are overshadowed by a large number of words correctly transcribed in target documents.

However, "speech-driven retrieval," where spoken queries are used to retrieve (textual) information, has not fully been explored, although it is related to numerous keyboard-less applications, such as telephone-based retrieval, car navigation systems, and user-friendly interfaces.

Unlike spoken document retrieval, speech-driven retrieval is still a challenging task, because recognition errors in short queries considerably decrease retrieval accuracy. A number of references addressing this issue can be found in past research literature.

Barnett et al. (1997) and Crestani (2000) independently performed comparative experiments related to speech-driven retrieval, where the DRAGON speech recognition system was used as an input interface for the INQUERY text retrieval system. They used as test queries 35 topics in the TREC collection, dictated by a single male speaker. However, these cases focused on improving text retrieval methods and did not address problems in improving speech recognition. As a result, errors in recognizing spoken queries (error rate was approximately $30 \%$ ) considerably decreased the retrieval accuracy.

Although we showed that the use of target document collections in producing language models for speech recognition significantly improved the performance of speech-driven retrieval (Fujii et al., 2002; Itou et al., 2001), a number of issues still remain open questions. 
Section 2 clarifies problems addressed in this paper. Section 3 overviews our speech-driven text retrieval system. Sections 4-6 elaborate on our methodology. Section 7 describes comparative experiments, in which an existing IR test collection was used to evaluate the effectiveness of our method. Section 8 discusses related research literature.

\section{Problem Statement}

One major problem in speech-driven retrieval is related to out-of-vocabulary (OOV) words.

On the one hand, recent IR systems do not limit the vocabulary size (i.e., the number of index terms), and can be seen as open-vocabulary systems, which allow users to input any keywords contained in a target collection. It is often the case that a couple of million terms are indexed for a single IR system.

On the other hand, state-of-the-art speech recognition systems still need to limit the vocabulary size (i.e., the number of words in a dictionary), due to problems in estimating statistical language models (Young, 1996) and constraints associated with hardware, such as memories. In addition, computation time is crucial for a real-time usage, including speech-driven retrieval. In view of these problems, for many languages the vocabulary size is limited to a couple of ten thousands (Itou et al., 1999; Paul and Baker, 1992; Steeneken and van Leeuwen, 1995), which is incomparably smaller than the size of indexes for practical IR systems.

In addition, high-frequency words, such as functional words and common nouns, are usually included in dictionaries and recognized with a high accuracy. However, those words are not necessarily useful for retrieval. On the contrary, low-frequency words appearing in specific documents are often effective query terms.

To sum up, the OOV problem is inherent in speech-driven retrieval, and we need to fill the gap between speech recognition and text retrieval in terms of the vocabulary size. In this paper, we propose a method to resolve this problem aiming at open-vocabulary speech-driven retrieval.

\section{System Overview}

Figure 1 depicts the overall design of our speechdriven text retrieval system, which consists of speech recognition, text retrieval and query completion modules. Although our system is currently implemented for Japanese, our methodology is language-independent. We explain the retrieval process based on this figure.

Given a query spoken by a user, the speech recognition module uses a dictionary and acoustic/language models to generate a transcription of the user speech. During this process, OOV words, which are not listed in the dictionary, are also detected. For this purpose, our language model includes both words and syllables so that OOV words are transcribed as sequences of syllables.

For example, in the case where "kankitsu (citrus)" is not listed in the dictionary, this word should be transcribed as /ka $\mathrm{N} \mathrm{ki} \mathrm{tsu/.} \mathrm{How-}$ ever, it is possible that this word is mistakenly transcribed, such as / $\mathrm{ka} \mathrm{N} \mathrm{ke} \mathrm{tsu/} \mathrm{and}$ /ka N ke tsu ke ko/.

To improve the quality of our system, these syllable sequences have to be transcribed as words, which is one of the central issues in this paper. In the case of speech-driven retrieval, where users usually have specific information needs, it is feasible that users utter contents related to a target collection. In other words, there is a great possibility that detected OOV words can be identified as index terms that are phonetically identical or similar.

However, since a) a single sound can potentially correspond to more than one word (i.e., homonyms) and b) searching the entire collection for phonetically identical/similar terms is prohibitive, we need an efficient disambiguation method. Specifically, in the case of Japanese, the homonym problem is multiply crucial because words consist of different character types, i.e., "kanji," "katakana," "hiragana," alphabets and other characters like numerals ${ }^{1}$.

To resolve this problem, we use a two-stage retrieval method. In the first stage, we delete OOV words from the transcription, and perform text retrieval using remaining words, to obtain a specific number of top-ranked documents according to the degree of relevance. Even if speech recognition is not perfect, these documents are potentially associated with the user speech more than the entire col-

\footnotetext{
${ }^{1}$ In Japanese, kanji (or Chinese character) is the idiogram, and katakana and hiragana are phonograms.
} 
lection. Thus, we search only these documents for index terms corresponding to detected OOV words.

Then, in the second stage, we replace detected OOV words with identified index terms so as to complete the transcription, and re-perform text retrieval to obtain final outputs. However, we do not re-perform speech recognition in the second stage.

In the above example, let us assume that the user also utters words related to "kankitsu (citrus)," such as "orenji (orange)" and "remon (lemon)," and that these words are correctly recognized as words. In this case, it is possible that retrieved documents contain the word "kankitsu (citrus)." Thus, we replace the syllable sequence/ka $\mathrm{N}$ ke tsu/ in the query with "kankitsu," which is additionally used as a query term in the second stage.

It may be argued that our method resembles the notion of pseudo-relevance feedback (or local feedback) for IR, where documents obtained in the first stage are used to expand query terms, and final outputs are refined in the second stage (Kwok and Chan, 1998). However, while relevance feedback is used to improve only the retrieval accuracy, our method improves the speech recognition and retrieval accuracy.

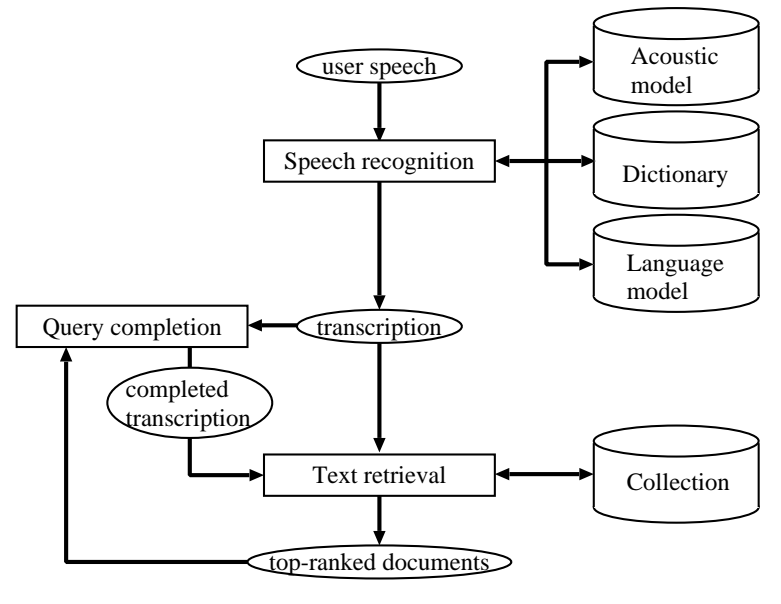

Figure 1: The overall design of our speech-driven text retrieval system.

\section{Speech Recognition}

The speech recognition module generates word sequence $W$, given phone sequence $X$. In a stochastic speech recognition framework (Bahl et al., 1983), the task is to select the $W$ maximizing $P(W \mid X)$, which is transformed as in Equation (1) through the Bayesian theorem.

$$
\arg \max _{W} P(W \mid X)=\arg \max _{W} P(X \mid W) \cdot P(W)
$$

Here, $P(X \mid W)$ models a probability that word sequence $W$ is transformed into phone sequence $X$, and $P(W)$ models a probability that $W$ is linguistically acceptable. These factors are usually called acoustic and language models, respectively.

For the speech recognition module, we use the Japanese dictation toolkit (Kawahara et al., 2000) ${ }^{2}$, which includes the "Julius" recognition engine and acoustic/language models. The acoustic model was produced by way of the ASJ speech database (ASJJNAS) (Itou et al., 1998; Itou et al., 1999), which contains approximately 20,000 sentences uttered by 132 speakers including the both gender groups.

This toolkit also includes development softwares so that acoustic and language models can be produced and replaced depending on the application. While we use the acoustic model provided in the toolkit, we use a new language model including both words and syllables. For this purpose, we used the "ChaSen" morphological analyzer ${ }^{3}$ to extract words from ten years worth of "Mainichi Shimbun" newspaper articles (1991-2000).

Then, we selected 20,000 high-frequency words to produce a dictionary. At the same time, we segmented remaining lower-frequency words into syllables based on the Japanese phonogram system. The resultant number of syllable types was approximately 700 . Finally, we produced a word/syllablebased trigram language model. In other words, OOV words were modeled as sequences of syllables. Thus, by using our language model, OOV words can easily be detected.

In spoken document retrieval, an open-vocabulary method, which combines recognition methods for words and syllables in target speech documents, was also proposed (Wechsler et al., 1998). However, this method requires an additional computation for recognizing syllables, and thus is expensive. In contrast, since our language model is a regular statistical $N$-gram model, we can use the same speech recognition framework as in Equation (1).

\footnotetext{
${ }^{2}$ http://winnie.kuis.kyoto-u.ac.jp/dictation

${ }^{3} \mathrm{http} / / /$ chasen.aist-nara.ac.jp
} 


\section{Text Retrieval}

The text retrieval module is based on the "Okapi" probabilistic retrieval method (Robertson and Walker, 1994), which is used to compute the relevance score between the transcribed query and each document in a target collection. To produce an inverted file (i.e., an index), we use ChaSen to extract content words from documents as terms, and perform a word-based indexing. We also extract terms from transcribed queries using the same method.

\section{Query Completion}

\subsection{Overview}

As explained in Section 3, the basis of the query completion module is to correspond OOV words detected by speech recognition (Section 4) to index terms used for text retrieval (Section 5). However, to identify corresponding index terms efficiently, we limit the number of documents in the first stage retrieval. In principle, terms that are indexed in topranked documents (those retrieved in the first stage) and have the same sound with detected OOV words can be corresponding terms.

However, a single sound often corresponds to multiple words. In addition, since speech recognition on a syllable-by-syllable basis is not perfect, it is possible that OOV words are incorrectly transcribed. For example, in some cases the Japanese word "kankitsu (citrus)" is transcribed as /ka $\mathrm{N}$ ke tsu/. Thus, we also need to consider index terms that are phonetically similar to OOV words. To sum up, we need a disambiguation method to select appropriate corresponding terms, out of a number of candidates.

\subsection{Formalization}

Intuitively, it is feasible that appropriate terms:

- have identical/similar sound with OOV words detected in spoken queries,

- frequently appear in a top-ranked document set,

- and appear in higher-ranked documents.

From the viewpoint of probability theory, possible representations for the above three properties include Equation (2), where each property corresponds to different parameters. Our task is to select the $t$ maximizing the value computed by this equation as the corresponding term for OOV word $w$.

$$
\sum_{d \in D_{q}} P(w \mid t) \cdot P(t \mid d) \cdot P(d \mid q)
$$

Here, $D_{q}$ is the top-ranked document set retrieved in the first stage, given query $q . P(w \mid t)$ is a probability that index term $t$ can be replaced with detected OOV word $w$, in terms of phonetics. $P(t \mid d)$ is the relative frequency of term $t$ in document $d . P(d \mid q)$ is a probability that document $d$ is relevant to query $q$, which is associated with the score formalized in the Okapi method.

However, from the viewpoint of empiricism, Equation (2) is not necessarily effective. First, it is not easy to estimate $P(w \mid t)$ based on the probability theory. Second, the probability score computed by the Okapi method is an approximation focused mainly on relative superiority among retrieved documents, and thus it is difficult to estimate $P(d \mid q)$ in a rigorous manner. Finally, it is also difficult to determine the degree to which each parameter influences in the final probability score.

In view of these problems, through preliminary experiments we approximated Equation (2) and formalized a method to compute the degree (not the probability) to which given index term $t$ corresponds to OOV word $w$.

First, we estimate $P(w \mid t)$ by the ratio between the number of syllables commonly included in both $w$ and $t$ and the total number of syllables in $w$. We use a DP matching method to identify the number of cases related to deletion, insertion, and substitution in $w$, on a syllable-by-syllable basis.

Second, $P(w \mid t)$ should be more influential than $P(t \mid d)$ and $P(d \mid q)$ in Equation (2), although the last two parameters are effective in the case where a large number of candidates phonetically similar to $w$ are obtained. To decrease the effect of $P(t \mid d)$ and $P(d \mid q)$, we tentatively use logarithms of these parameters. In addition, we use the score computed by the Okapi method as $P(d \mid q)$.

According to the above approximation, we compute the score of $t$ as in Equation (3).

$$
\sum_{d \in D_{q}} P(w \mid t) \cdot \log (P(t \mid d) \cdot P(d \mid q))
$$


It should be noted that Equation (3) is independent of the indexing method used, and therefore $t$ can be any sequences of characters contained in $D_{q}$. In other words, any types of indexing methods (e.g., word-based and phrase-based indexing methods) can be used in our framework.

\subsection{Implementation}

Since computation time is crucial for a real-time usage, we preprocess documents in a target collection so as to identify candidate terms efficiently. This process is similar to the indexing process performed in the text retrieval module.

In the case of text retrieval, index terms are organized in an inverted file so that documents including terms that exactly match with query keywords can be retrieved efficiently.

However, in the case of query completion, terms that are included in top-ranked documents need to be retrieved. In addition, to minimize a score computation (for example, DP matching is time-consuming), it is desirable to delete terms that are associated with a diminished phonetic similarity value, $P(w \mid t)$, prior to the computation of Equation (3). In other words, an index file for query completion has to be organized so that a partial matching method can be used. For example, /ka N ki tsu/ has to be retrieved efficiently in response to $/ \mathrm{ka} \mathrm{N}$ ke tsu/.

Thus, we implemented a forward/backward partial-matching method, in which entries can be retrieved by any substrings from the first/last characters. In addition, we index words and word-based bigrams, because preliminary experiments showed that $\mathrm{OOV}$ words detected by our speech recognition module are usually single words or short phrases, such as "ozon-houru (ozone hole)."

\section{Experimentation}

\subsection{Methodology}

To evaluate the performance of our speech-driven retrieval system, we used the IREX collection ${ }^{4}$. This test collection, which resembles one used in the TREC ad hoc retrieval track, includes 30 Japanese topics (information need) and relevance assessment (correct judgement) for each topic, along with target

\footnotetext{
${ }^{4}$ http://cs.nyu.edu/cs/projects/proteus/irex/index-e.html
}

documents. The target documents are 211,853 articles collected from two years worth of "Mainichi Shimbun" newspaper (1994-1995).

Each topic consists of the ID, description and narrative. While descriptions are short phrases related to the topic, narratives consist of one or more sentences describing the topic. Figure 2 shows an example topic in the SGML form (translated into English by one of the organizers of the IREX workshop).

However, since the IREX collection does not contain spoken queries, we asked four speakers (two males/females) to dictate the narrative field. Thus, we produced four different sets of 30 spoken queries. By using those queries, we compared the following different methods:

1. text-to-text retrieval, which used written narratives as queries, and can be seen as a perfect speech-driven text retrieval,

2. speech-driven text retrieval, in which only words listed in the dictionary were modeled in the language model (in other words, the OOV word detection and query completion modules were not used),

3. speech-driven text retrieval, in which OOV words detected in spoken queries were simply deleted (in other words, the query completion module was not used),

4. speech-driven text retrieval, in which our method proposed in Section 3 was used.

In cases of methods 2-4, queries dictated by four speakers were used independently. Thus, in practice we compared 13 different retrieval results. In addition, for methods 2-4, ten years worth of Mainichi Shimbun Japanese newspaper articles (1991-2000) were used to produce language models. However, while method 2 used only 20,000 high-frequency words for language modeling, methods 3 and 4 also used syllables extracted from lower-frequency words (see Section 4).

Following the IREX workshop, each method retrieved 300 top documents in response to each query, and non-interpolated average precision values were used to evaluate each method. 


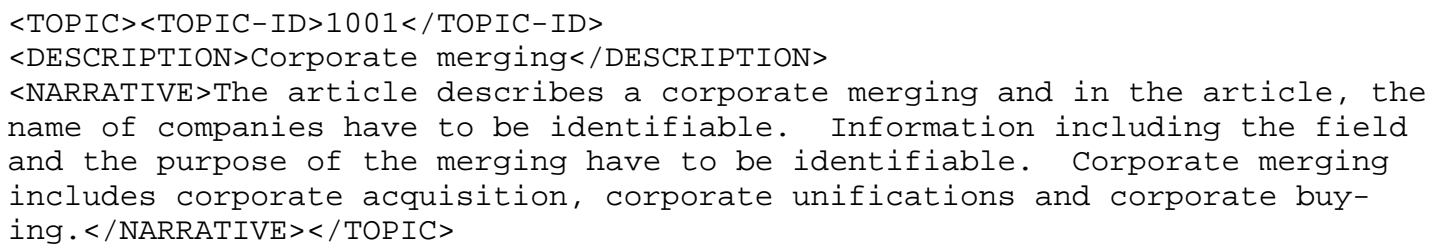

Figure 2: An English translation for an example topic in the IREX collection.

\subsection{Results}

First, we evaluated the performance of detecting OOV words. In the 30 queries used for our evaluation, 14 word tokens (13 word types) were OOV words unlisted in the dictionary for speech recognition. Table 1 shows the results on a speaker-byspeaker basis, where "\#Detected" and "\#Correct" denote the total number of OOV words detected by our method and the number of OOV words correctly detected, respectively. In addition, "\#Completed" denotes the number of detected OOV words that were corresponded to correct index terms in 300 top documents.

It should be noted that "\#Completed" was greater than "\#Correct" because our method often mistakenly detected words in the dictionary as OOV words, but completed them with index terms correctly. We estimated recall and precision for detecting OOV words, and accuracy for query completion, as in Equation (4).

$$
\begin{aligned}
\text { recall } & =\frac{\# \text { Correct }}{14} \\
\text { precision } & =\frac{\# \text { Correct }}{\# \text { Detect }} \\
\text { accuracy } & =\frac{\# \text { Completed }}{\# \text { Detect }}
\end{aligned}
$$

Looking at Table 1, one can see that recall was generally greater than precision. In other words, our method tended to detect as many OOV words as possible. In addition, accuracy of query completion was relatively low.

Figure 3 shows example words in spoken queries, detected as OOV words and correctly completed with index terms. In this figure, OOV words are transcribed with syllables, where ":" denotes a long vowel. Hyphens are inserted between Japanese words, which inherently lack lexical segmentation.
Second, to evaluate the effectiveness of our query completion method more carefully, we compared retrieval accuracy for methods 1-4 (see Section 7.1). Table 2 shows average precision values, averaged over the 30 queries, for each method ${ }^{5}$. The average precision values of our method (i.e., method 4) was approximately $87 \%$ of that for text-to-text retrieval.

By comparing methods 2-4, one can see that our method improved average precision values of the other methods irrespective of the speaker. To put it more precisely, by comparing methods 3 and 4, one can see the effectiveness of the query completion method. In addition, by comparing methods 2 and 4 , one can see that a combination of the OOV word detection and query completion methods was effective.

It may be argued that the improvement was relatively small. However, since the number of OOV words inherent in 30 queries was only 14 , the effect of our method was overshadowed by a large number of other words. In fact, the number of words used as query terms for our method, averaged over the four speakers, was 421 . Since existing test collections for IR research were not produced to explore the OOV problem, it is difficult to derive conclusions that are statistically valid. Experiments using larger-scale test collections where the OOV problem is more crucial need to be further explored.

Finally, we investigated the time efficiency of our method, and found that CPU time required for the query completion process per detected OOV word was 3.5 seconds (AMD Athlon MP 1900+). However, an additional CPU time for detecting OOV words, which can be performed in a conventional speech recognition process, was not crucial.

\footnotetext{
${ }^{5}$ Average precision is often used to evaluate IR systems, which should not be confused with evaluation measures in Equation (4).
} 
Table 1: Results for detecting and completing OOV words.

\begin{tabular}{lcccccc}
\hline \hline Speaker & \#Detected & \#Correct & \#Completed & Recall & Precision & Accuracy \\
\hline Female \#1 & 51 & 9 & 18 & 0.643 & 0.176 & 0.353 \\
Female \#2 & 56 & 10 & 18 & 0.714 & 0.179 & 0.321 \\
Male \#1 & 33 & 9 & 12 & 0.643 & 0.273 & 0.364 \\
Male \#2 & 37 & 12 & 16 & 0.857 & 0.324 & 0.432 \\
\hline Total & 176 & 40 & 64 & 0.714 & 0.226 & 0.362 \\
\hline
\end{tabular}

\begin{tabular}{|c|c|c|}
\hline OOV words & Index terms (syllables) & $\overline{\text { English gloss }}$ \\
\hline $\begin{array}{l}\text { /gu re : pu ra chi na ga nol } \\
\text { /ya yo i chi tal } \\
\text { /ni ku ku ra i sul } \\
\text { /be N pil }\end{array}$ & $\begin{array}{l}\text { gureepu-furuutsu/gu re : pu fu ru : tsul } \\
\text { Yayoi-jidai/ya yo i ji da i/ } \\
\text { nikku-puraisu/ni q ku pu ra i sul } \\
\text { benpi/be N pi/ }\end{array}$ & $\begin{array}{l}\text { grapefruit } \\
\text { the Yayoi period } \\
\text { Nick Price } \\
\text { constipation }\end{array}$ \\
\hline
\end{tabular}

Figure 3: Example words detected as OOV words and completed correctly by our method.

\subsection{Analyzing Errors}

We manually analyzed seven cases where the average precision value of our method was significantly lower than that obtained with method 2 (the total number of cases was the product of numbers of queries and speakers).

Among these seven cases, in five cases our query completion method selected incorrect index terms, although correct index terms were included in topranked documents obtained with the first stage. For example, in the case of the query 1021 dictated by a female speaker, the word "seido (institution)" was mistakenly transcribed as /se $\mathrm{N}$ do/. As a result, the word "sendo (freshness)," which is associated with the same syllable sequences, was selected as the index term. The word "seido (institution)" was the third candidate based on the score computed by Equation (3). To reduce these errors, we need to enhance the score computation.

In another case, our speech recognition module did not correctly recognize words in the dictionary, and decreased the retrieval accuracy.

In the final case, a fragment of a narrative sentence consisting of ten words was detected as a single OOV word. As a result, our method, which can complete up to two word sequences, mistakenly processed that word, and decreased the retrieval accuracy. However, this case was exceptional. In most cases, functional words, which were recognized with a high accuracy, segmented OOV words into shorter fragments.
Table 2: Non-interpolated average precision values, averaged over 30 queries, for different methods.

\begin{tabular}{lcccc}
\hline \hline Speaker\Method & 1 & 2 & 3 & 4 \\
\hline Female \#1 & - & 0.2831 & 0.2834 & 0.3195 \\
Female \#2 & - & 0.2745 & 0.2443 & 0.2846 \\
Male \#1 & - & 0.3005 & 0.2987 & 0.3179 \\
Male \#2 & - & 0.2787 & 0.2675 & 0.2957 \\
\hline Total & 0.3486 & 0.2842 & 0.2734 & 0.3044 \\
\hline
\end{tabular}

\section{Related Work}

The method proposed by Kupiec et al. (1994) and our method are similar in the sense that both methods use target collections as language models for speech recognition to realize open-vocabulary speech-driven retrieval.

Kupiec et al's method, which is based on word recognition and accepts only short queries, derives multiple transcription candidates (i.e., possible word combinations), and searches a target collection for the most plausible word combination. However, in the case of longer queries, the number of candidates increases, and thus the searching cost is prohibitive. This is a reason why operational speech recognition systems have to limit the vocabulary size.

In contrast, our method, which is based on a recent continuous speech recognition framework, can accept longer sentences. Additionally, our method uses a two-stage retrieval principle to limit a search space in a target collection, and disambiguates only detected OOV words. Thus, the computation cost can be minimized. 


\section{Conclusion}

To facilitate retrieving information by spoken queries, the out-of-vocabulary problem in speech recognition needs to be resolved. In our proposed method, out-of-vocabulary words in a query are detected by speech recognition, and completed with terms indexed for text retrieval, so as to improve the recognition accuracy. In addition, the completed query is used to improve the retrieval accuracy. We showed the effectiveness of our method by using dictated queries in the IREX collection. Future work would include experiments using larger-scale test collections in various domains.

\section{References}

Lalit. R. Bahl, Frederick Jelinek, and Robert L. Mercer. 1983. A maximum likelihood approach to continuous speech recognition. IEEE Transactions on Pattern Analysis and Machine Intelligence, 5(2):179-190.

J. Barnett, S. Anderson, J. Broglio, M. Singh, R. Hudson, and S. W. Kuo. 1997. Experiments in spoken queries for document retrieval. In Proceedings of Eurospeech97, pages 1323-1326.

Fabio Crestani. 2000. Word recognition errors and relevance feedback in spoken query processing. In Proceedings of the Fourth International Conference on Flexible Query Answering Systems, pages 267-281.

Atsushi Fujii, Katunobu Itou, and Tetsuya Ishikawa. 2002. Speech-driven text retrieval: Using target IR collections for statistical language model adaptation in speech recognition. In Anni R. Coden, Eric W. Brown, and Savitha Srinivasan, editors, Information Retrieval Techniques for Speech Applications (LNCS 2273), pages 94-104. Springer.

John S. Garofolo, Ellen M. Voorhees, Vincent M. Stanford, and Karen Sparck Jones. 1997. TREC-6 1997 spoken document retrieval track overview and results. In Proceedings of the 6th Text REtrieval Conference, pages 83-91.

K. Itou, M. Yamamoto, K. Takeda, T. Takezawa, T. Matsuoka, T. Kobayashi, K. Shikano, and S. Itahashi. 1998. The design of the newspaper-based Japanese large vocabulary continuous speech recognition corpus. In Proceedings of the 5th International Conference on Spoken Language Processing, pages 32613264.

Katunobu Itou, Mikio Yamamoto, Kazuya Takeda, Toshiyuki Takezawa, Tatsuo Matsuoka, Tetsunori Kobayashi, and Kiyohiro Shikano. 1999. JNAS:
Japanese speech corpus for large vocabulary continuous speech recognition research. Journal of Acoustic Society of Japan, 20(3):199-206.

Katunobu Itou, Atsushi Fujii, and Tetsuya Ishikawa. 2001. Language modeling for multi-domain speechdriven text retrieval. In IEEE Automatic Speech Recognition and Understanding Workshop.

Pierre Jourlin, Sue E. Johnson, Karen Spärck Jones, and Philip C. Woodland. 2000. Spoken document representations for probabilistic retrieval. Speech Communication, 32:21-36.

T. Kawahara, A. Lee, T. Kobayashi, K. Takeda, N. Minematsu, S. Sagayama, K. Itou, A. Ito, M. Yamamoto, A. Yamada, T. Utsuro, and K. Shikano. 2000. Free software toolkit for Japanese large vocabulary continuous speech recognition. In Proceedings of the 6th International Conference on Spoken Language Processing, pages 476-479.

Julian Kupiec, Don Kimber, and Vijay Balasubramanian. 1994. Speech-based retrieval using semantic co-occurrence filtering. In Proceedings of the ARPA Human Language Technology Workshop, pages 373377.

K.L. Kwok and M. Chan. 1998. Improving two-stage adhoc retrieval for short queries. In Proceedings of the 21st Annual International ACM SIGIR Conference on Research and Development in Information Retrieval, pages 250-256.

Douglas B. Paul and Janet M. Baker. 1992. The design for the Wall Street Journal-based CSR corpus. In Proceedings of DARPA Speech \& Natural Language Workshop, pages 357-362.

S.E. Robertson and S. Walker. 1994. Some simple effective approximations to the 2-poisson model for probabilistic weighted retrieval. In Proceedings of the 17th Annual International ACM SIGIR Conference on Research and Development in Information Retrieval, pages 232-241.

Herman J. M. Steeneken and David A. van Leeuwen. 1995. Multi-lingual assessment of speaker independent large vocabulary speech-recognition systems: The SQALE-project. In Proceedings of Eurospeech95, pages 1271-1274.

Martin Wechsler, Eugen Munteanu, and Peter Schäuble. 1998. New techniques for open-vocabulary spoken document retrieval. In Proceedings of the 21st Annual International ACM SIGIR Conference on Research and Development in Information Retrieval, pages 2027.

Steve Young. 1996. A review of large-vocabulary continuous-speech recognition. IEEE Signal Processing Magazine, pages 45-57, September. 